\title{
On the Quantum Theory of Impact Phenomenon for the Conditions of Elastic Deformation of Impacted Body
}

\author{
Zdzisław Pluta, Tadeusz Hryniewicz* \\ Koszalin University of Technology, Raclawicka 15-17, PL 75-620 Koszalin, Poland \\ *E-mail address: Tadeusz.Hryniewicz@tu.koszalin.pl
}

„If one has just got a hammer every problem seems to remind you the nail".

Abraham Maslow

\begin{abstract}
The work contains some of the elements of adequate quantum theory of impact phenomenon. These elements form the prelude to verification of the existent non-adequate theory of the impact phenomenon. At first a correct definition of impact has been formulated. For comparison, the interpretation of the elastic impact according to the existent knowledge is given. Then, taking advantage of the new correctly formulated the principle of mechanical energy conservation, the energetic states of impacting body have been described. Basing on this, the formula on dynamic deflection has been derived. This formula is adequate in character and fully corresponds with investigated reality.
\end{abstract}

Keywords: Impact; Quantum (potential); Potential field; Energy; Principle of mechanical energy conservation; Space-time; Dynamics

\section{INTRODUCTION}

In the frameworks of introduction it is worth underlining the title name of the theory of impact phenomenon this work has been devoted to. This is a quantum theory and such a notation was introduced on purpose. One would like to stress that it does not refer only to the theories describing microscopic reality. A quantum change in energy takes place also in macroscopic systems which reflect the presented here theory.

Quantum refers simply to the potential, i.e. a measure of energy, that is a portion of this magnitude. One should admit that these portions refer to the potential behaviours of a body between consecutive energy states. These states are marked on the neighbouring potential fields.

The use of properly formulated principle of mechanical energy conservation on these fields is the key to solve the impact problem that is the dynamic deformation of impacted material. This paper is to present just an adequate cognition way. 


\section{SPOTS OF IMPACT PHENOMENON OCCURRENCE}

Impact occurs during operating of a variety of devices and installations. Operating of most machines, especially in case of to-and-fro motion machines, is associated with impacts. Sometimes the impacts occur in consequence of clearance appearance, where the elements are connected, and often the impact loadings accompany inevitably with a normal work of machine (smithy/forging machines, pneumatic drills/hammers).

The strength calculations of elements of that kind of machines are to take into account the impact aspects. Stresses arising inside these elements, as well as their deformations, will be many times higher than in case of their static loadings only. Selection of a material for such elements is quite an essential problem for materials engineers.

Therefore at first this phenomenon should be recognized, described, and formulated quantitatively. This phenomenon, as being considerably complex, has not been recognized fully. The analysis of references of the subject, presented in the next Chapter, is to reveal what drawbacks are characteristic for the existent impact theory. First of all there is lack of description of this phenomenon, and it appears that there are still no proper cognition methods applied. It appears, the classical approach to the considered problem is not enough now.

Impact results not only from the characteristics of particular machines. It is also the essence of the machining process itself. Plastic treatment, especially free forging, is a good example to illustrate the occurrence of impact as a necessary condition of desired material deformations.

The impact phenomenon is used also to determine a specified measure of material hardness. One of them is Shore method in which the measure of hardness is bound height of a weight ended with a steel sphere or diamond cone, falling down from a determined height on the studied material. It is known that the Shore sclerometer serves for the purpose. Also a Poldi hardness tester, as a comparative method, is based on the impact phenomenon.

Impact is a phenomenon the material resistance against it is investigated. The resistance of a material to impact is called the impact strength. The Charpy pendulum machine is used to this kind of durability/strength research.

The impact occurs also during materials treatment by means of a high-pressure waterabrasive jet as well as the abrasive jet only. Apart from the others, the problem of mapping of curvilinear trajectory in the machining zone, as well as evaluation of the surface roughness formed with this method, occur here [1]. According to the reference [1], the equation of this trajectory corresponds with the logarithmic spiral. It also says that in the references, e.g. in [2, 3 ] there is an attempt to equalize the trajectory by parabola or by means of a polynomial of higher degrees.

Given examples of descriptions of the determined strength investigations and machining processes where the phenomenon of impact occurs indicate that the problem is not so well known. The Authors reject the up-to-date classical tools. These tools are not used to solve the problems because they rather overshadow a proper solution. Therefore some new tools are created. These new tools allow for different thinking about the impact problem with the result being its final solution. Included at the beginning the aphorism is referred just to the contents of last paragraph. Let this hammer-nail link of the cognition process (Fig. 1) be some sort of the warning sign on the way to cognition of the natural reality. 


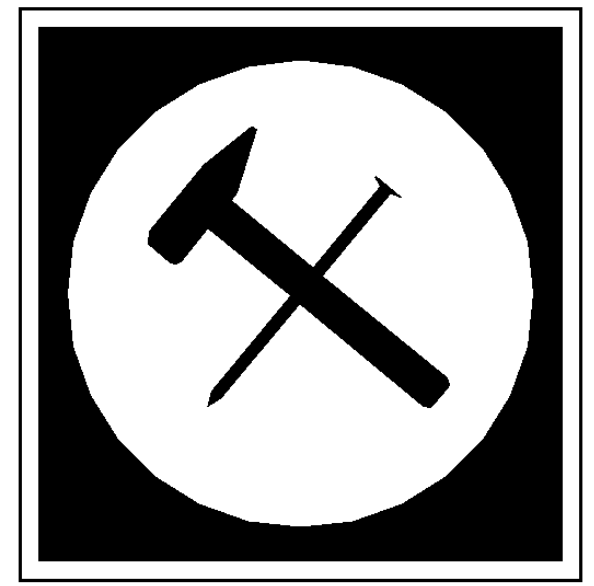

Fig. 1. Hammer-nail link of the cognition process.

\section{CRITICAL ANALYSIS OF LITERATURE INFORMATION ABOUT IMPACT}

Of the impact phenomenon just an enigmatic information is found. To study the category of mechanical phenomena bearing in mechanics the name of impact, the method being the theorem of impulses is still applied. The impact is called as [4]: „Such a phenomenon, during which in a very short time (e.g. in time equaling to few thousandth parts of a second) the amount of motion of material system obtains an increment/increase not very small one but of the same order as the amount of motion of the system".

It is worth remaining at this paragraph to analyze critically some things. Let some terminological questions be the subject of this analysis. The classical mechanics says about the amount of motion of a material system by referring this notion to the momentum of the system. What for is that unnecessary erroneous terminological structure? Though motion is a mind magnitude [5] so it is immeasurable unlike in case of physical magnitudes. Thus one cannot say about the amount of motion. The category of amount concerns the magnitudes which possess units, that is the magnitudes of physical nature.

The next terminological question of which the essence is analogous and contradicts to the rule of monomiality [6] requires that only one name means that notion. It concerns the impulse for which the synonym is, according to the existent knowledge, time-effect. There are notions of theorem of impulses or momentum, or sometimes of momentum and time-effects.

It appears the impulse is quite different than time-effect, because impulse is the derivative of acceleration, whereas the time-effect is the derivative of inertia force. Such a differentiation of magnitudes is noted at a proper description of variable motion or dynamics of a material body. That is not possible in the frames of classical mechanics and this is why the time-effect is determined apparently/artificially. It is done by mathematical transformation of a formula on inertia force, this formula being the second Newton's law. One may add that so called the d'Alembert's principle was formed by the same way... Quo vadis, science?

As to the definition of impact, it does not explain anything. The amount of motion of a system obtains such increment as the amount of a system, or impetus of a system gains such increase as the system impetus. No clarification! Vicious circle!

A critical analysis is required concerning this fragment of existent knowledge on the impact phenomenon. This critics is to revel reasons of the scientific impossibility as to the quantitative description of the impact. Further it is said that the impulse theorem does not 
allow to determine the variability of instantaneous force in time. Hence the character of this function is unknown.

Here again the notion of an instantaneous force appears. The instantaneous force is the force referred to one instant of time, so one cannot consider it in time (or collection/set of instants). It is simply a thrust/opposition force of the deformed material, or contact force.

The classical theory of collision of solid bodies, created by a series of investigators, beginning from Galilei, and finishing on Newton, assumed the collided bodies as ideally rigid and the process of collision as momentary. That theory enabled only to determine effects of collision, i.e. change of velocity of the colliding solids, or internal veracities of the impact phenomenon. Time of its duration, magnitudes of the contact forces and deformations remained completely non-clarified.

The Hertz theory partly solves this problem. It is based on the hypothesis that relation between the contact force and spot deformation of the bodies at impact is the same as at their static compression. In fact this hypothesis is equivalent with avoiding of the inertia force. Therefore experimental check/proof of this theory revealed only a satisfactory agreement of calculation and experimental data. However, it is too little!

Based on the literature one could develop the impact phenomenon. Let us finish these consideration by referring to [7] where further scientific developments related to the discussed subject are presented. However, the analysis of the literature indicates that there is no exact solution, as to the quantitative description, of the impact problem. For the solution, the principle of mechanical energy conservation (properly understood, i.e. other than up-to-date classical one), will be used. This newly formed principle says that if an undone work is added to the done gravitational work, the planned work is obtained.

Just a few words about Hertz theory: this relation between the force and deformation during impact is really the same as at the static contact of material bodies. That is known but does not mean the neglecting of inertia forces. These forces, as well as other forces being the measure of interaction of the bodies during impact, are independent from each other. They are to be considered separately and closer explained.

\section{DEFINITION OF IMPACT PHENOMENON}

Impact has not been properly defined as yet that is evident in the presented critical literature analysis of the subject. It was not possible because even description of the subject phenomenon has erroneous structure and does not explain the essence. Therefore the existent classical theory of impact is not adequate and does not refer to the investigated reality.

Impact is a phenomenon of dynamic character (where is the dynamics, the impact occurs). The problem is the dynamics has not got a proper interpretation. The existent science, based on the so called d'Alembert's principle accepted as a paradigm, does not describe the variable motion (this motion proves of the body dynamics, and not the mass of the body). All these solutions of differential equations, constituted in accordance to the d'Alembert's principle, do not correspond with the real motion but are rather descriptions of fictitious/ apparent motions. This principle, as itself, is fictitious in nature [8].

A general definition of the impact phenomenon should be as follows: "Impact is the phenomenon of dynamic deformation of material bodies". This definition corresponds with any rules obligatory at creation of all definitions [6]. Of course, one could formulate the definition in a way the dynamics notion would not be opened but only with a determined explanation, e.g. the dynamics takes place in space-times only, that is between potential fields. Therefore the impact definition may be formulated as follows: "Impact is the 
phenomenon of deformation of material bodies in space-times". The notion of space-times, potential fields, as well as the classification of the former ones are presented in references [9$11]$.

Now one could develop this general definition by formulating some detailed definitions what could mean a parallel creation of classification of the impact phenomenon. Therefore, taking into account the classification discriminant, being the state of aggregation of a material body, one should introduce and define the notion of: mechanical, hydraulic, pneumatic... impact. Therefore the definition of mechanical impact should be: "Mechanical impact is the dynamic phenomenon of deformation of solids".

The next classification criterion is, e.g. the type of deformation of impacted body (treated as the rigid body, or a solid), or elastic, plastic, elasto-plastic deformation. Then going further, more detailed definitions should be given, e.g. the elastic mechanical impact would be defined as: "Elastic mechanical impact is a phenomenon of dynamic deformation of a solid, with the deformation being elastic in character".

The graphical illustration of this classification of impact phenomenon is presented in Fig. 2. However, the mechanical impact is to be considered, without further developments of other types of impacts. The Fig. 2 contains some exemplified branches of classification of the impact phenomenon.

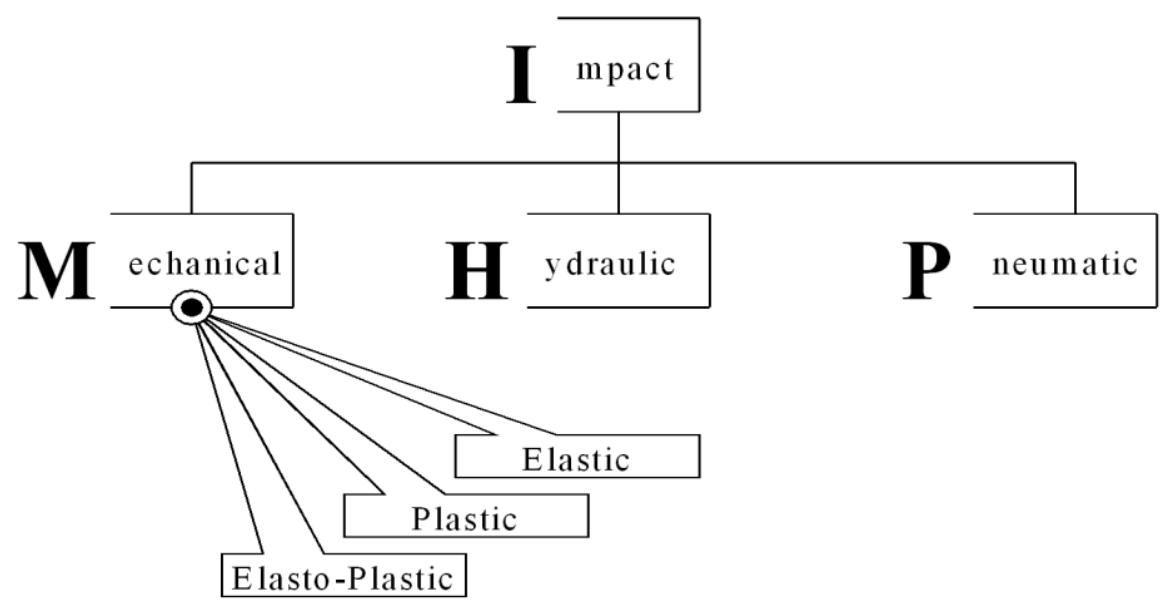

Fig. 2. Classification of the impact phenomenon.

\section{INTERPRETATION OF THE ELASTIC IMPACT ACCORDING TO EXISTENT KNOWLEDGE}

Earlier described the classical theorem of impulses does not allow to describe the course of impact nor even its terminal parameters. Of impact itself one judges only based on a determined relation between the velocities of bodies before impact and after it (that is about restitution coefficient).

To determine dependence between the dynamic and static deflections of an elastic element, blown by a determined rigid body, the so called principle of energy conservation is commonly used. It is usually assumed that a mass of ideally elastic element is negligibly small in relation to the mass of hitting body. Such a specific system will be analyzed here: 
according to the existent classical knowledge, and then using a new revolutionary impact theory, being adequate in its character.

Let the body of weight $Q$ (Fig. 3) rests at vertically situated spring of the rigidity coefficient $k$ (here a specific mechanical rigidity, or elasticity is considered, so at the same time one may assume the elasticity coefficient). The elastic deflection of the element with the static deflection $h_{s}$ has occurred.

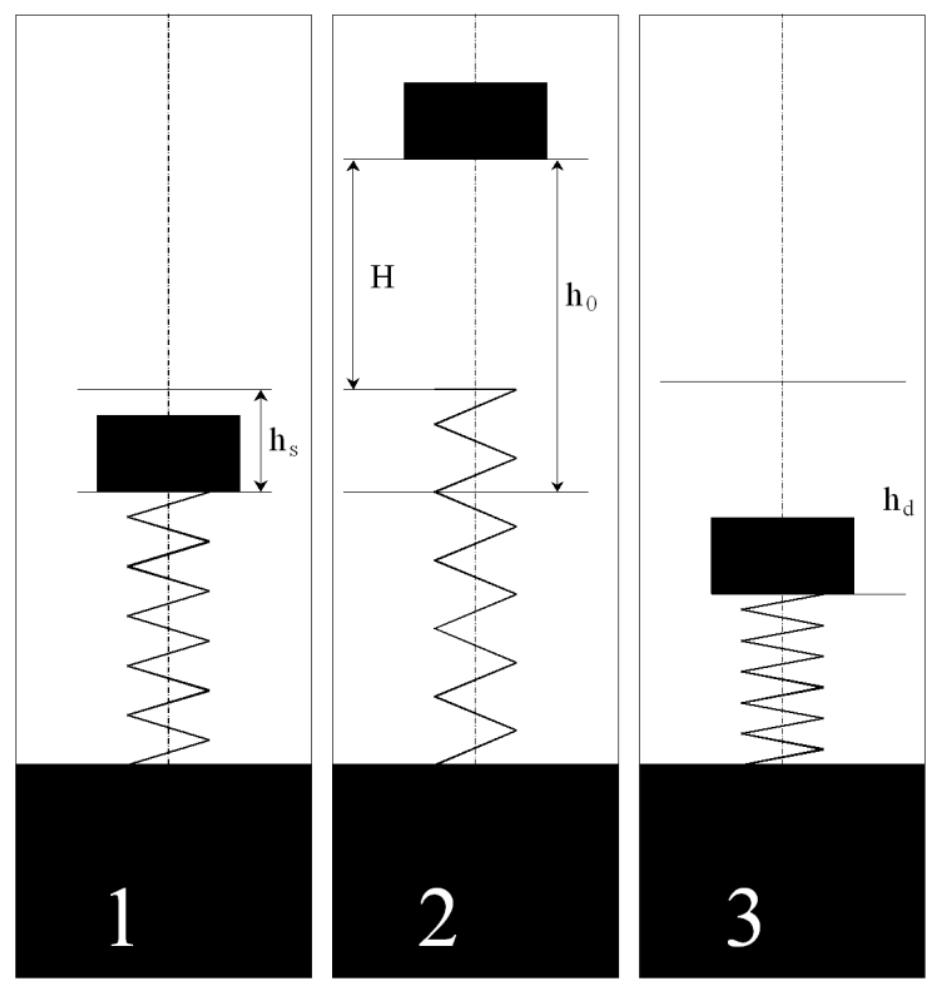

Fig. 3. Energetic states of a body referred to the impact phenomenon.

In the next step, this body is lifted to the height $h_{o}$. That resulted the primarily compressed spring returned to the free initial state. The distance between this weight and a free frontal surface of spring, denoted by symbol $H$, forms (together with the system parameter $h_{o}$ ) the characteristics of the second system state.

In the next step the hitting body (the third system state) falls down, hits the spring and deflects it. This time the deflection, as the dynamic one $h_{d}$, is respectively bigger than the primary static deflection.

No force is marked here, no particular potential field is marked nor named, i.e. no system characteristics adequate to its real behaviours has been introduced. The attempt was made to keep the classic existent course of argumentation/reasoning in the form of natural still respected one. The three described situations have been separated only, by the numbers $(1,2$, 3), which correspond to the static energetic states of a material body, with first two states being temporary and the third, instantaneous one. The temporary states may last for a determined series/sequence of moments/instants. The instantaneous state means it lasts just one moment. Further on, these energetic aspects will be discussed.

The so called principle of energy conservation was used. The stages of such directed creative actions result from the indicative scheme of particular forces courses (Fig. 4). According to the classical interpretation of reality they are the following forces: gravity force 
$Q$ and the elasticity force $S$. Both forces have been presented in function of the path/way length $h$ of the material body.

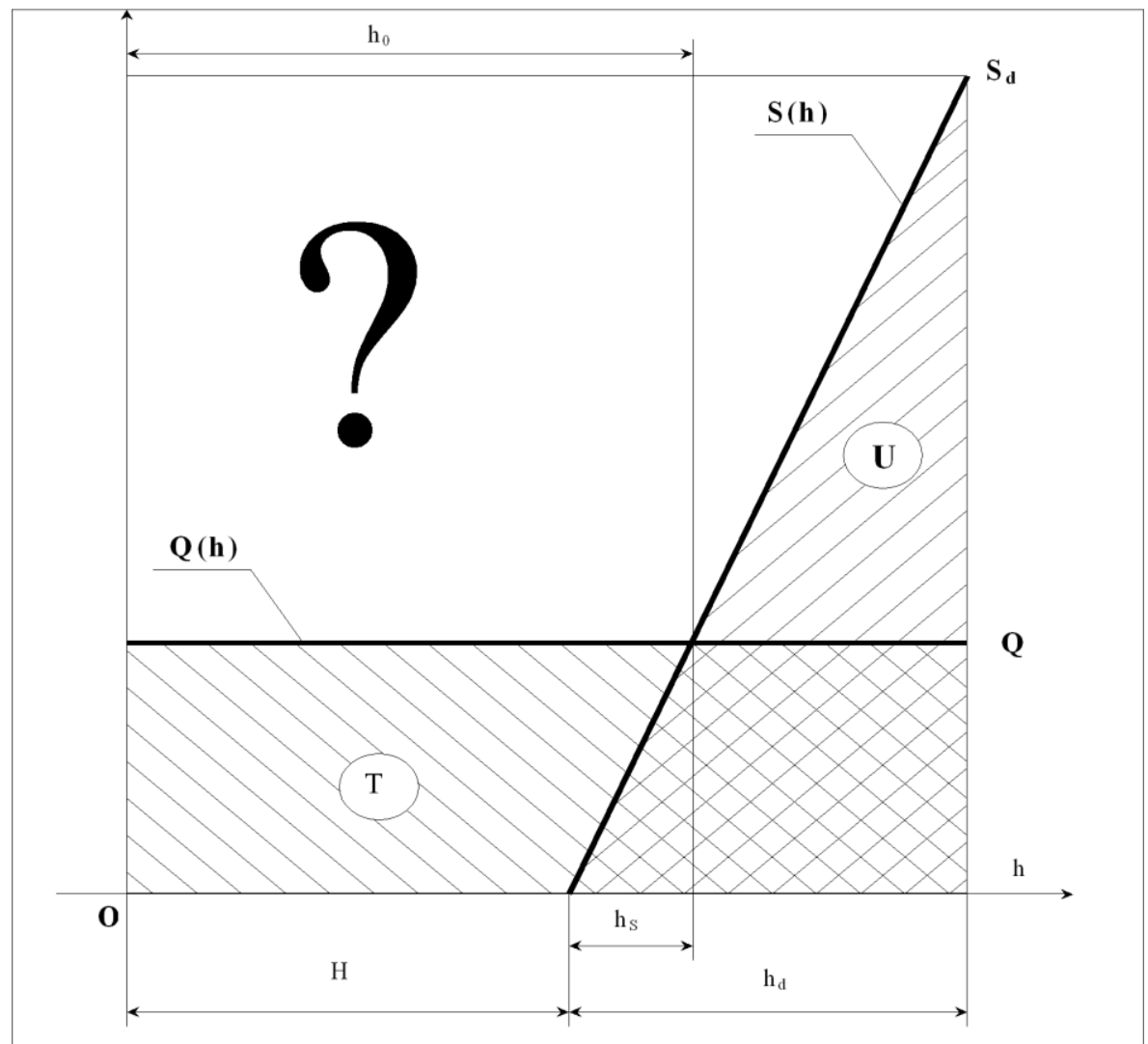

Fig. 4. The course of the so called potential energy and "deformation energy".

According to the mentioned principle, loss of potential energy of the weight $Q$, which equals:

$$
T=Q\left(H+h_{d}\right)
$$

is equal the energy of deformation gained by the elastic element. That former energy is equal:

$$
U=\frac{S_{d} h_{d}}{2}
$$

where the symbol $S_{d}$ denotes the dynamic force of elasticity.

Taking into account that:

$$
S_{d}=k h_{d}
$$

the so called elastic energy of deformation is written by the following formula:

$$
U=\frac{k h_{d}^{2}}{2}
$$


Therefore, by comparing formulae (1) and (4), the following form of the conservation principle is obtained:

$$
Q\left(H+h_{d}\right)=\frac{k h_{d}^{2}}{2}
$$

and further on, because of:

$$
Q=k h_{s}
$$

it assumes the following configuration:

$$
k h_{s}\left(H+h_{d}\right)=\frac{k h_{d}^{2}}{2}
$$

whereas developed, takes this form:

$$
h_{d}^{2}-2 h_{s} h_{d}-2 h_{s} H=0
$$

After solution of the equation, due to $h_{d}$, one obtains:

$$
h_{d}=h_{s} \pm \sqrt{h_{s}^{2}+2 h_{s} H}
$$

assuming the positive solution, as negative (-) has no physical sense. Therefore, finally:

$$
h_{d}=h_{s}+\sqrt{h_{s}^{2}+2 h_{s} H}
$$

By analyzing the last dependence one may determine $h_{d}$ also for $H=0$. Then $h_{d}=2 h_{s}$. One may admit, that is a pity no physical sense of the whole reasoning has been given by taking care of such a sense at the end of the path/way.

In the last picture (Fig. 4) there are hatched areas which correspond with the so called potential energy of the weight, as well as "deformation energy". The apparent in Fig. 4 the interrogative sign means the existent grounded knowledge forms much room for a doubt. Putting aside the discussion on arising terminological subjects one has to state that science says about the equality of works: gravitational and elastic one, respectively. They are no energies nor their changes.

One should ask, where is the inertia force of the body. Hence the analyzed system contains three bodies: earth, spring, and the body impacting the spring. Therefore three forces should be considered: gravity force $Q$, elasticity force $S$, and inertia force $B$ (so many forces as bodies). The last one has been omitted.

One should admit there cannot be equality of works in such a body motion, that is in the free motion. Even after taking into account the inertia force, there will not be equality in the particular works. The equality occurs but in the forced motion. It touches the works done: by determined bodies and over the determined bodies. Therefore the impact phenomenon may be properly described only by using properly understood the principle of mechanical energy conservation. 


\section{ADEQUATE PRINCIPLE OF MECHANICAL ENERGY CONSERVATION IN USE FOR DESCRIPTION OF THE IMPACT PHENOMENON}

The mechanical energy, the principle of conservation of this type of energy, and impact phenomenon are to be considered. In [12], the sense of inertia notion was presented. Before the conservation principle is presented here, in reference to the impact phenomenon description, it is worth presenting the definitions of inertia and mechanical energy $[12,13]$.

"The inertia of a body is its susceptibility/tendency to take the most advantageous its state, that is stable one": this definition of inertia indicates clearly the sense/turn of measure of this magnitude. This force will be always directed into the stable state of the body.

„The mechanical energy is the energy of solid", and this is the definition of that magnitude with the potential as the measure. It is one of many types of energies, a fundamental magnitude with the following definition, as: "The energy of a body is its ability to perform a work at transition to the neighbouring potential field".

The principle of energy conservation (energy measures or potentials) says that the sum of all energies on all potential fields is unchanged and constant [12]. Thus the general notation of that principle possesses the following form:

$$
\sum_{i=1}^{n} V_{i}=i d e m
$$

The grounded, common, classical, so called principle of the mechanical energy conservation has nothing to do with the properly understood the mechanical energy. Telling the truth, the existent knowledge in this subject may be eventually treated as the principle of summation of the gravitation work in the variable motion cycle. Symbol $V_{i}$ of that description means the measure of a non-determined energy, $i$ - means the potential number, and $n$-is the number of all potentials.

More detailed notation of the presented principle, referred to the particular potential fields $(0,1,2, \ldots, j)$, has the following configuration:

$$
\sum_{i=1}^{n_{0}} V_{i}^{(0)}=\sum_{i=1}^{n_{1}} V_{i}^{(1)}=\ldots=\sum_{i=1}^{n_{j}} V_{i}^{(j)}=i d e m
$$

Here the sign $j$ denotes the number of consecutive potential field, and symbol $n_{j}$ - number of potentials referred to this field.

Coming to description of the impact phenomenon, at first it is worth determining the energetic state of the system under a forced motion. This will allow to know the relations occurring between particular forces and the gravity force. Then, after taking to analysis the system in a free motion referred to the impact, this knowledge will be needed.

When a system is in a stable state (Fig. 5), then the inertia force $B_{o}$ is the biggest one and co-exists with the gravity force $Q$. The state of co-existence is denoted by a symbol $\otimes$, then $Q \otimes B_{o}$. The static equilibrium of the system is recorded here by the following equality: $Q=S_{o}$, where $S_{o}$ means the initial elasticity force. From that the former record does not result that any energetic state (here, a stable state) takes place simultaneously. Not before, the extended record $\left(Q \otimes B_{o}=S_{o}\right)$ reveals that mystery at full length. 


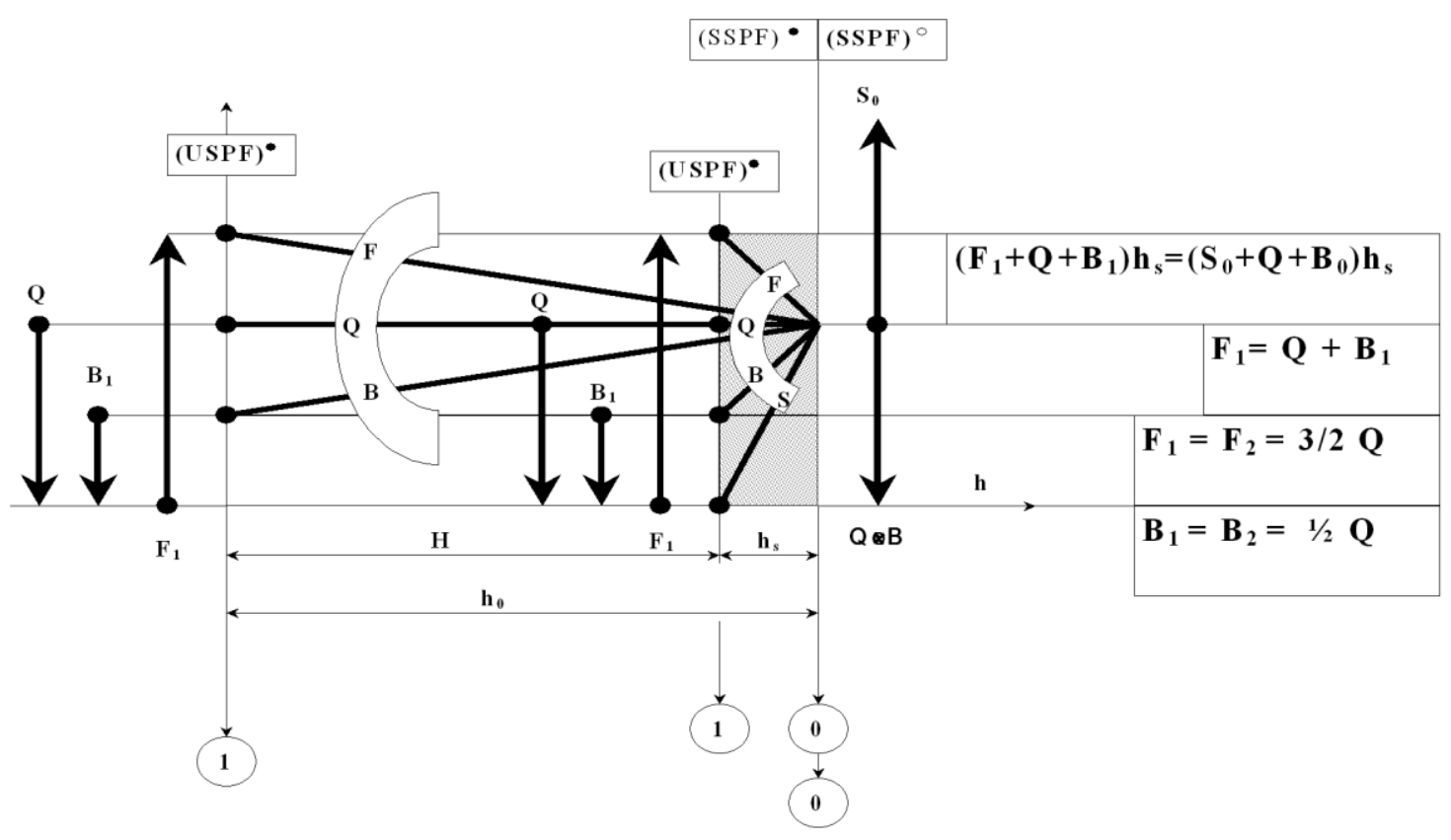

Fig. 5. Courses of particular forces under variable forced motion.

After these explanations the principle of energy conservation on initial field, zero potential field, may be recorded as:

$$
V_{S}+V_{Q}+V_{B}=i d e m
$$

Its more exact form may be presented as follows:

$$
\left(S_{0}+Q+B_{0}\right) h_{s}=S_{0} h_{s}+Q h_{s}+B_{0} h_{s}=i d e m
$$

The particular symbols mean here: $V_{S}$ - elastic potential, $V_{Q}$ - gravitation potential, $V_{B}-$ inertia potential, $B_{o}$ - initial inertia force, or the inertia intensity of potential field, respectively. Thus $S_{o}$ is elastic intensity, and $Q$ - gravitation intensity of this field.

It is worth presenting in more detail the characteristics of this potential field [14-22]. This is a temporary stable static potential field (SSPF) ${ }^{\bullet}$, so the whole system is in temporary stable static energetic field. The following energies are on this field: elastic, gravitation, and inertia.

To replace the body on a neighbouring, first potential field, some external stimulus should be used. Let it be a biologic body (a palm). In a moment, being on instantaneous stable static potential field (SSPF) ${ }^{\circ}$, that stimulus introduces system to the space-time determined by the length $h_{\mathrm{s}}$ (dotted area). In this area, there are four different bodies in action, i.e. the biologic body, earth, subjected body, and an elastic element. The course of forces, interacting one on another, is different in character that is apparent from the analyzed Fig. 5.

On the next, or first as marked, potential field, temporary and unstable static potential field (USPF) ${ }^{\bullet}$, there is no elastic energy. It has been replaced here by the biologic energy. The 
principle of sum energy conservation (their measures, or potentials) on this field has the following form:

$$
V_{F}+V_{Q}+V_{B}=i d e m
$$

where $V_{F}$ denotes the biologic potential.

A detailed record of that energetic equilibrium has the following structure:

$$
\left(F_{1}+Q+B_{1}\right) h_{s}=F_{1} h_{s}+Q h_{s}+B_{1} h_{s}=i d e m
$$

where $F_{1}$ denotes a biologic force (intensity of the biologic first potential field), $B_{1}-$ is the inertia force on this field, or its inertia intensity. The gravitation intensity - worth to admit has not undergone to a change.

By connecting the formulae (14), (16), one obtains the equation in the form:

$$
\left(S_{0}+Q+B_{0}\right) h_{s}=\left(F_{1}+Q+B_{1}\right) h_{s}
$$

and - after taking into account, $S_{o}=Q \otimes B_{o}$, a contraction of the equation by $h_{s}$ and some other operations - the following record is obtained:

$$
F_{1}+B_{1}=2 Q
$$

Taking into account further equilibria of the system on the first field (force equilibrium), recorded this way:

$$
F_{1}=Q+B_{1}
$$

leads to the system of equations (18), (19), of which the solution - due to $F_{1}$ and $B_{1}$ - gives the results:

$$
F_{1}=\frac{3}{2} Q \quad B_{1}=\frac{1}{2} Q
$$

Transmission of the body to another, a higher positioned potential field, as seen in Fig. 5 , will not result in a change of equilibrium of all forces. Therefore the same relations will be taking place there as well.

Let us consider now a free motion of the body with an impact phenomenon that happened at some time. The body will be first carried up at height $H$ against a free surface of spring where $S=0$ (Fig. 6). Released from the palm press/squeeze, the body will be at first moment on an instantaneous unstable static potential field (USPF) ${ }^{\circ}$, and further it will be passing through the consecutive $(0,1,2, \ldots)$ - also instantaneous potential fields. 


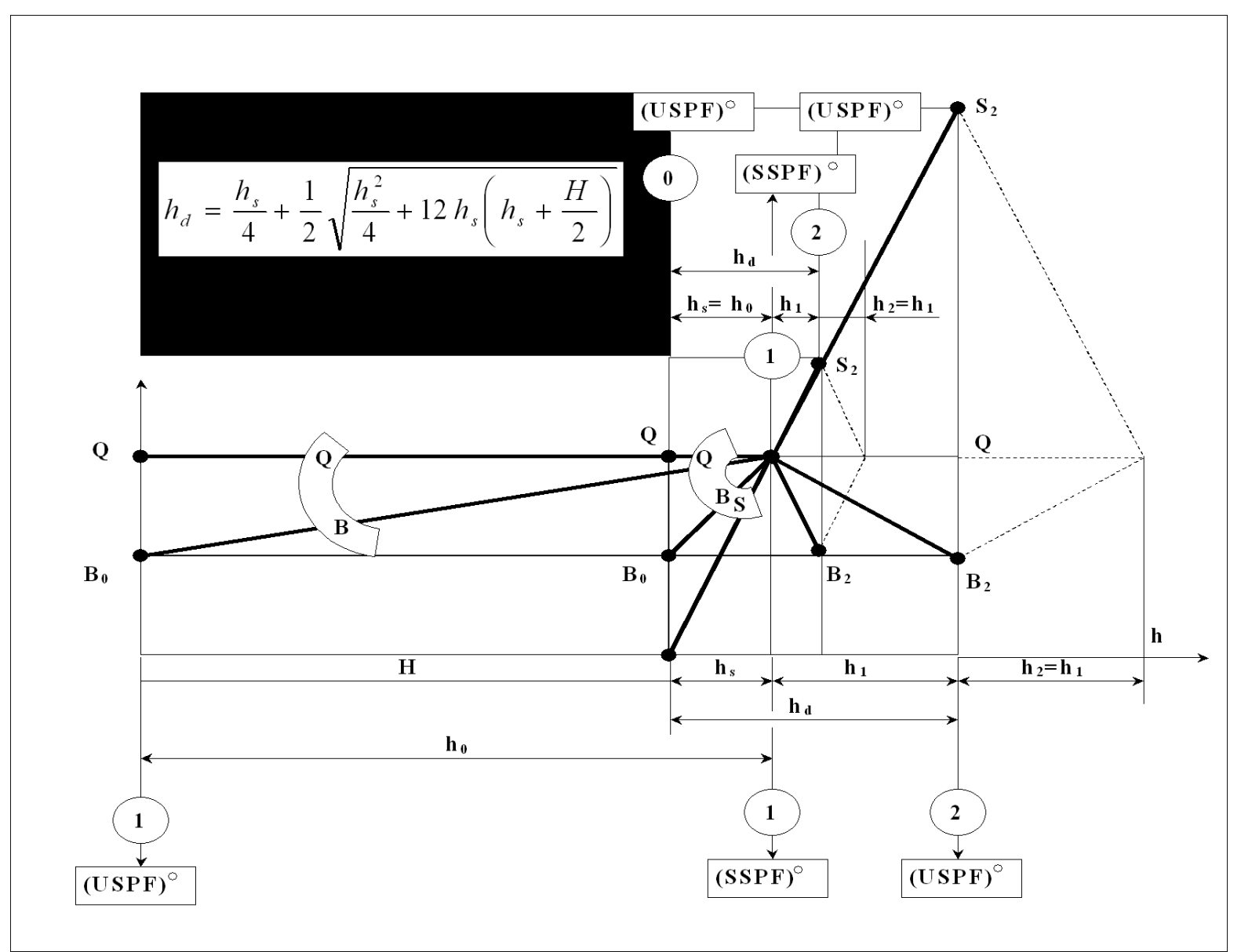

Fig. 6. Courses of forces in a free motion, corresponding with impact phenomenon.

The principle of energy conservation on three consecutive potential fields will be analyzed. Such a limitation results from the goal presented here: derivation of an adequate formula on dynamic deflection. In connection with this, the courses of forces for a space-time being situated between these fields, have been marked in Fig. 6 (solid line). In addition, the courses of forces for only one of the consecutive space-times have been introduced (dashed line).

At first this principle for zero - initial (0) and second (2) potential fields has been formulated. Then the attention will be concentrated on the first (1) field where for $H>0$ this kind of energy does not occur.

The principle of conservation of all instantaneous mechanical energy on the mentioned first two potential fields has the following form:

$$
V_{Q}^{(0)}+V_{B}^{(0)}=V_{Q}^{(2)}+V_{B}^{(2)}+V_{S}^{(2)}
$$

and after more detailed approach:

$$
Q \cdot h_{0}+B_{0} \cdot h_{0}=S_{2} \cdot h_{2}+Q \cdot h_{2}+B_{2} \cdot h_{2}
$$

where $h_{o}$ denotes length of the first/initial, and $h_{2}-$ of third space-time. 
Due to the equality of neighbouring space-times: second and third $\left(h_{1}=h_{2}\right)$ - one could treat the same record as the law of threshold moment lever, as it was done earlier in [13]. In this case only the first sum of moments corresponds with potentials, and the right side, recorded as: $S_{2} h_{1}+Q h_{1}+B_{2} h_{1}$ - would mean the sum of threshold moments.

Knowing that $B_{o}=B_{1}=B_{2}=Q: 2$, that was pointed out earlier (see formula (20)), taking into account also:

$$
\begin{gathered}
h_{0}=H+h_{s} \\
h_{2}=h_{1}=h_{d}-h_{s} \\
Q=k h_{s} \\
S_{2}=k\left(h_{s}+h_{1}\right)=k h_{d}
\end{gathered}
$$

after doing some mathematical operations, has been obtained finally:

$$
h_{d}^{2}+\frac{1}{2} h_{s} h_{d}-3 h_{s}\left(h_{s}+\frac{1}{2} H\right)=0
$$

Now this equation should be solved and a positive result taken in view of getting the dependence searched between dynamic and static deflections of the elastic element. And this is:

$$
h_{d}=-\frac{h_{s}}{4}+\frac{1}{2} \sqrt{\frac{h_{s}^{2}}{4}+12 h_{s}\left(h_{s}+\frac{H}{2}\right)}
$$

This is a final mathematic form of the principle of mechanical energy conservation. It may be treated as its specific conclusion coming out of the subject principle, recorded in a general form (21). If in the frames of solution analysis, $H=0$, is substituted, one obtains $h_{d}=$ $1.5 h_{s}$, and not $h_{d}=2 h_{s}$ as it is in the existent theory. One should stress, that the first result has been confirmed by the experiment, and this is of great importance. Also the dependence (28) has been confirmed by an experiment. That polarization of results, coming out of the improper classical existent description of reality, should draw an attention of scientists especially in view of further development of science.

It is worth determining this energy (internal potential $U^{*}$ ) on the second field, marked with symbol 1. Then one may formulate the principle of energy behaviour for the first two potential fields. It is presented as:

$$
Q \cdot h_{0}+B_{0} \cdot h_{0}=Q \cdot h_{1}+B_{1} \cdot h_{1}+S_{1} \cdot h_{1}+U^{*}
$$

Having in mind that $B_{o}=0.5 Q, B_{1}=S_{l}=Q, h_{o}=H+h_{s}, h=h_{d}-h_{s}, Q=k h_{s}$, one obtains as a result:

$$
\frac{3}{2} k h_{s}\left(H+h_{s}\right)=3 k h_{s}\left(h_{d}-h_{s}\right)+U^{*}
$$

what after solving, due to $U^{*}$, gives: 


$$
U^{*}=\frac{9}{2} k h_{s}^{2}-3 k h_{s}\left(h_{d}+\frac{1}{2} H\right)
$$

For $H=0$, or the situation determined by dependence $h_{d}=1.5 h_{s}$, the internal energy potential of this energy $U^{*}=0$.

\section{CONCLUSION}

Presented above the adequate description of a portion of energetic reality indicates on urgent need to verify the existent non-adequate theory of impact phenomenon. The range of problems connected with this phenomenon is quite large. That proper dependence between dynamic and static deflections has been just derived. It is concerned only with the first cycle of impact but there are more cycles.

Further energetic states of the considered system have not been described yet. Also the courses of all forces in particular space-times have to be described. Temporal courses of these magnitudes also have not been determined. It is necessary to present temporary characteristics - courses in time of such magnitudes which are the kinetic characteristics of this mass-elastic system.

One could indicate some other magnitudes that should be determined in the frames of the in-depth analysis of impact phenomenon. All these have so big cognition space that one cannot place them in one article, or even a cycle of this type of papers. A separate book is needed with the proposed title as e.g. "Quantum theory of impact phenomenon".

It is not the end, because also descriptions of other phenomena also should be verified, and there are lots of them. Summing up, one should state that the classical mechanics requires a general verification which could allow to formulate adequate in fact descriptions of the reality. They are generally perspective tasks but they are worth noticing at present, this way throwing light on further path for the cognition of natural reality.

\section{References}

[1] E. Wantuch, R. Kot, Problems of accuracy and curvilinear mapping in high-pressure abrasive-water-jet treatment. Proceedings of XXVI Scientific School of Abrasive Treatment, Łódź, September 2003, pp. 329-337.

[2] A. Momber, R. Kovacevic, ASME Manufacturing Science and Engineering 68(1) (1994) 361-366.

[3] A. Momber, R. Kovacevic, Principles of Abrasive Water Jet Machining. Sprinper Verlag Berlin Heilderberg New York, 1998.

[4] J. Buchholz, J. Leyko, General mechanics. Dynamics (in Polish). PWN, Warszawa-Łódź 1966.

[5] Zdzisław Pluta, Normalizacja 9 (1994) 10-11.

[6] M. Mazur, Technical terminology (in Polish), WNT, Warszawa 1961.

[7] S. D. Ponomariev (ed.), Contemporary methods of strength calculations in the machine building. Dynamic loadings (in Polish), PWN, Warszawa 1957. 
[8] Zdzisław Pluta, $L A B 6$ (2004) 42-46.

[9] Zdzisław Pluta, NIT (Nauka, Innowacje, Technika) 5-6 (2004) 58-61.

[10] Zdzisław Pluta, Tadeusz Hryniewicz, Journal of Quantum Information Science 1 (2011) 127-134.

[11] Zdzisław Pluta, Tadeusz Hryniewicz, Journal of Quantum Information Science 1 (2011) 149-160.

[12] Zdzisław Pluta, LAB (Laboratoria, Aparatura, Badania) 2 (2004) 50-52.

[13] Zdzisław Pluta, $L A B 1$ (2005) 50-54.

[14] Zdzisław Pluta, Tadeusz Hryniewicz, Int. J. Adv. Manuf. Technol. 42 (2009) 515-522.

[15] Zdzisław Pluta, Tadeusz Hryniewicz, Int. J. Adv. Manuf. Technol. 43 (2009) 440-448.

[16] Zdzisław Pluta, Tadeusz Hryniewicz, Int. J. Adv. Manuf. Technol. 51 (2010) 35-43, DOI: $10.1007 / \mathrm{s} 00170-010-2595-5$

[17] Zdzisław Pluta, Tadeusz Hryniewicz, Int. J. Adv. Manuf. Technol. 62(5) (2012) 529-542.

[18] Zdzisław Pluta, Tadeusz Hryniewicz, Tribology Trans. 55(2) (2012) 230-236.

[19] Zdzisław Pluta, Tadeusz Hryniewicz, International Letters of Chemistry, Physics and Astronomy 2 (2013) 49-59.

[20] Zdzisław Pluta, Tadeusz Hryniewicz, International Letters of Chemistry, Physics and Astronomy 3 (2013) 67-84.

[21] Pluta Z., Hryniewicz T., International Letters of Chemistry, Physics and Astronomy 4 (2013) 56-72.

[22] Pluta Z., Hryniewicz T., International Letters of Chemistry, Physics and Astronomy 6 (2013) 116-136. 